\title{
Analysis of stellar populations with large empirical libraries at high spectral resolution
}

\author{
Philippe Prugniel ${ }^{1,2} \dagger$, Mina Koleva ${ }^{1,3}$, Pierre Ocvirk ${ }^{4}$, \\ Damien Le Borgne ${ }^{4}$ and Caroline Soubiran ${ }^{5}$ \\ ${ }^{1}$ Université Lyon 1, Observatoire de Lyon, St. Genis Laval, F-69230, France; CNRS UMR5574 \\ ${ }^{2}$ Observatoire de Paris, GEPI, F-75014, France \\ ${ }^{3}$ Department of Astronomy, St. Kl. Ohridski University of Sofia, BG-1164 Sofia, Bulgaria \\ ${ }^{4}$ CEA Saclay/Service d'Astrophysique, Gif-sur-Yvette Cedex, F-91191, France \\ ${ }^{5}$ Observatoire de Bordeaux, F-33270, Floirac, France
}

\begin{abstract}
The stellar population models dramatically progressed with the arrival of large and complete libraries, ELODIE, CFLIB (=Indo-US) and MILES at a relatively high resolution. We show that the quality of the fits is not anymore limited by the size of the stellar libraries in a large range of ages ( 0.1 to $10 \mathrm{Gyr})$ and metallicities $(-2$ to $+0.4 \mathrm{dex})$. The main limitations of the empirical stellar libraries are (i) the coverage of the parameter space (lack of hot stars of low metallicity), (ii) the precision and homogeneity of the atmospheric parameters and (iii) the non-resolution of individual element abundances (in particular $[\alpha / \mathrm{Fe}]$ ). Detailed abundances measurements in the large libraries, and usage of theoretical libraries are probably the next steps, and we show that a combination between an empirical (ELODIE) and a theoretical library (Coelho et al. 2005) immediately improves the modeling of ( $\alpha$-enhanced) globular clusters.
\end{abstract}

Keywords. galaxies: abundances; globular clusters: general

\section{High precision analysis of stellar populations}

The new empirical libraries that became available in the last 5 years allowed dramatic improvements in the analysis of stellar populations. The ELODIE library (Prugniel \& Soubiran 2001, 2004; a new version, 3.1, will be released soon) contains 2000 spectra of 1400 stars at high spectral resolution $(0.55 \AA \approx R=10000$ and $R=42000)$ in the wavelength range 390 to $680 \mathrm{~nm}$. The CFLIB (or Indo US) library (Valdes et al. 2004) have a lower resolution (about $1 \AA$ ) but an extended wavelength coverage 346 to $946 \mathrm{~nm}$. It contains 1273 stars but suffers from a poor flux calibration. MILES (Sánchez-Blázquez 2006), finally, has a slightly lower resolution $(2.3 \AA)$ and narrower wavelength range (352 to $750 \mathrm{~nm}$ ) but has been carefully flux-calibrated for 985 stars. The coverage of the parameters space is comparable for the three libraries.

The increase of spectral resolution is a noticeable characteristics. In the past, population models, like Pegase (Fioc \& Rocca-Volmerange 1997) were suited for multi-band photometry or low resolution spectra. With the new models, population analysis becomes sensitive to the depth and shape of the spectral features. As shown in Koleva et al. (2006) the optimal resolution, in term of observing time required to get a given precision on the kinematics, age or metallicity, is of the order of the physical broadening of the object. For giant elliptical galaxies a $\mathrm{R}=400$ is sufficient, but for a globular cluster $R=40000$ would be an advantage. In practice, a resolution of $R=10000$ is well suited for 


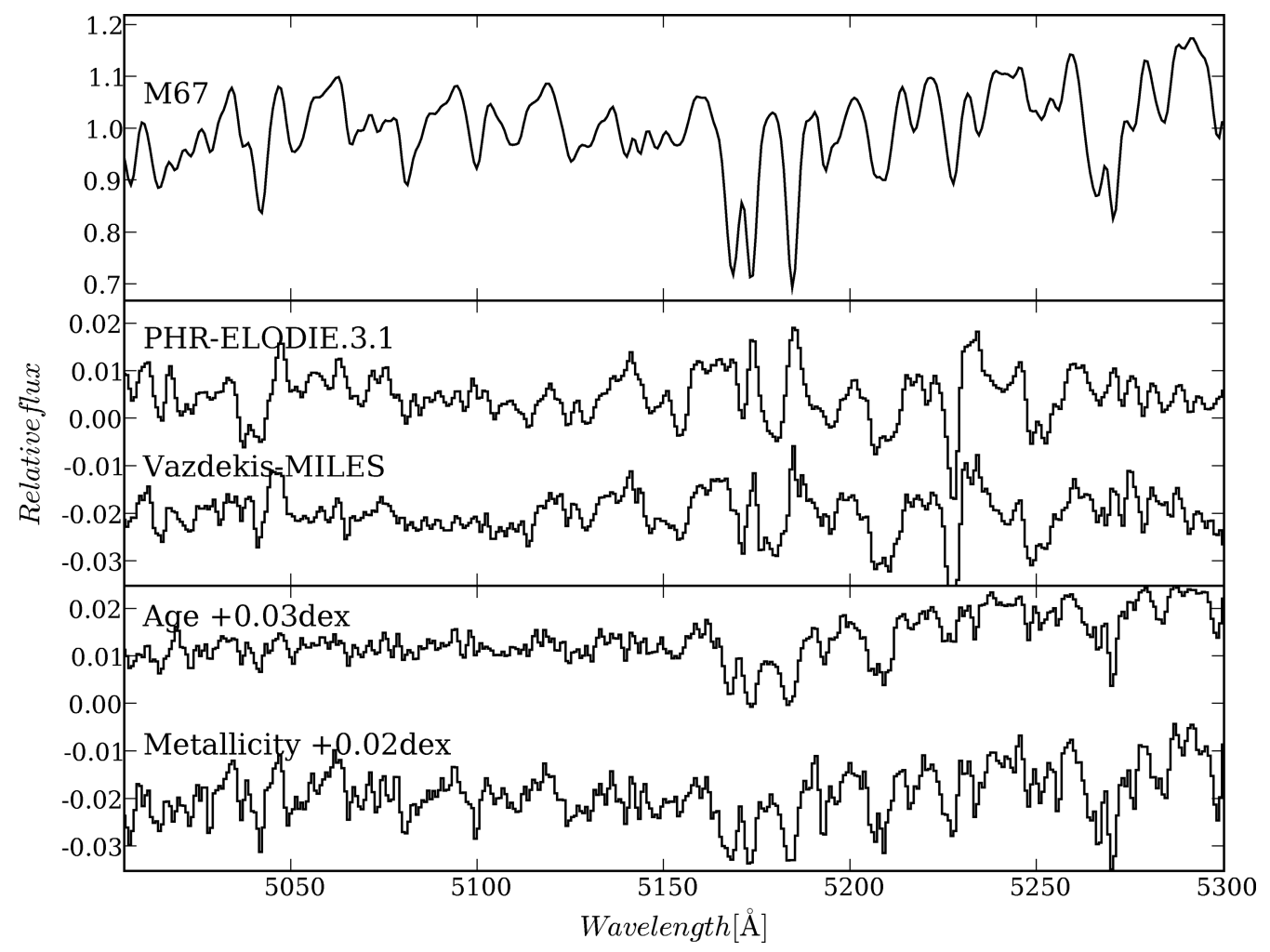

Figure 1. Example of population fit to the spectrum of M67. Top panel: M67 spectrum (from Schiavon et al. 2004) shown as reference. Middle panel: residuals to the best fit with Pegase.HR and Vazdekis models, the vertical scale is magnified by a factor 10. Bottom panel: Effect of increasing the age by 0.03 dex $(7.5 \%)$ or the metallicity by 0.02 dex $(5 \%)$ in the Pegase.HR-ELODIE.3.1 models.

the usual setups available on the spectrographs (model should have higher resolution than the spectrograph in order to inject the instrumental broadening in the model prior to the analysis). To benefit of the high resolution, methods performing full spectrum fitting simultaneously of the kinematics and characteristics of the populations have been developed (Ocvirk et al. 2006; Chilingarian et al., this conference).

Koleva et al. (posters at this conference) have shown the remarkable consistency between models independently built with these library: Pegase.HR using ELODIE and Vazdekis using MILES, and the reliability of the determination of the characteristics of the stellar populations using full spectrum fitting. Figure 1 gives an example of fit to M67 (observations from Schiavon et al. 2004) with Vazdekis/MILES and Pegase.HR/ELODIE. The quality of the fit is unprecedentedly good and the parameters of the population match well the CMD determinations. The residuals to the two fits ( $\mathrm{rms} \approx 0.5 \%$ ) are very similar, and the same exercise repeated on other observations shows that they are dominated by (i) the noise and errors in the observations and (ii) physical features, like the misfit on $\mathrm{Mg}$ and $\mathrm{CN}$ due to the different abundances in the clusters and in the library. 


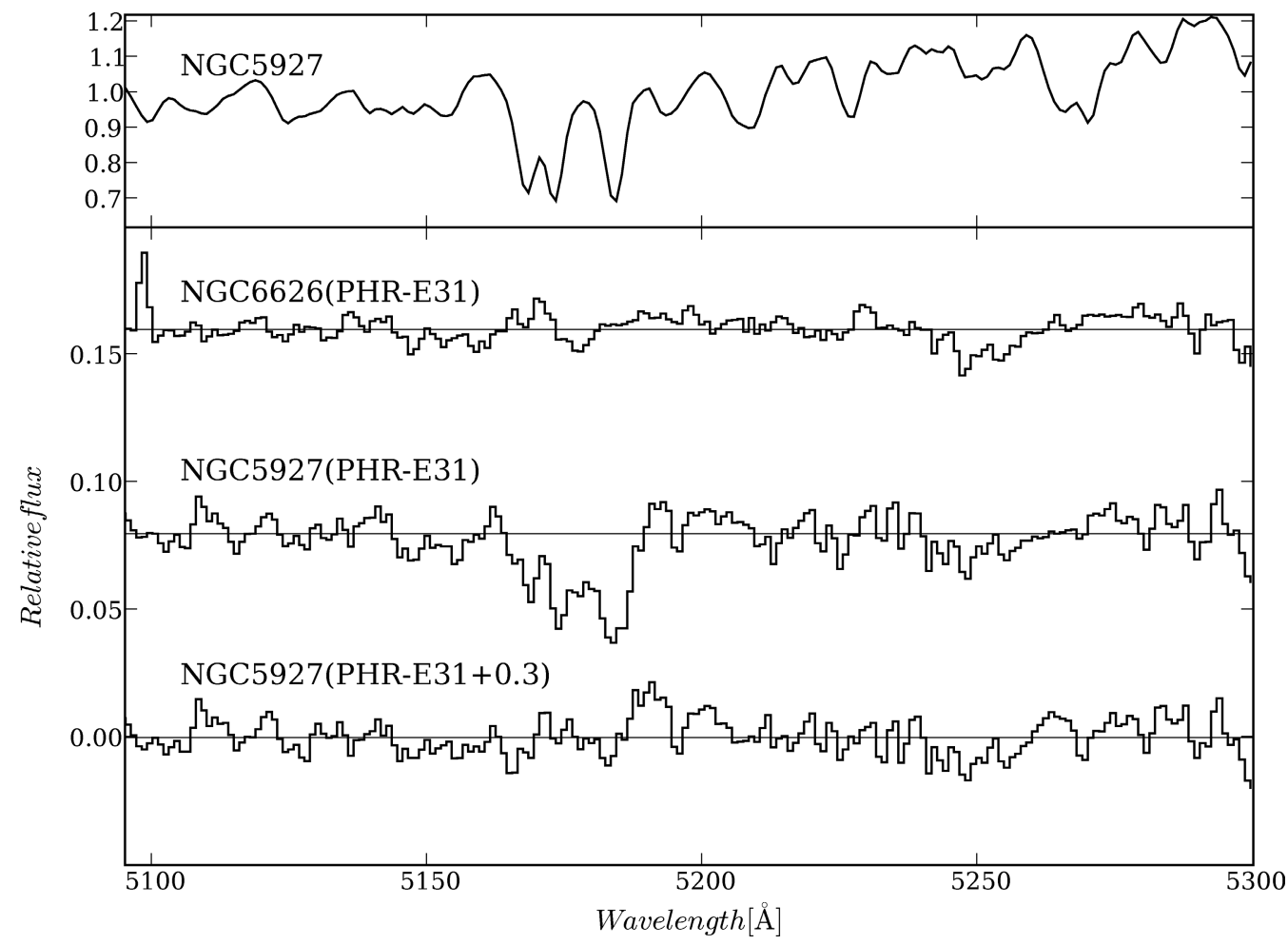

Figure 2. Population fit of the globular clusters NGC 5927 (high metallicity) and NGC 6626 (low metallicity). Top: Spectrum of NGC 5927 (from Schiavon et al. 2005) shown as reference. Bottom: (a) Residuals from the best-fit standard Pegase.HR-ELODIE.3.1 models for NGC 6626 and (b) for NGC 5927. The latter shows important mismatch on $\mathrm{Mg}$, due $\mathrm{Mg} / \mathrm{Fe}$ enhancement relative to the library. (c) Residuals for NGC 5927 to the semi-empirical models where the ELODIE spectra are differentially corrected for $[\alpha / \mathrm{Fe}]$ abundance using Coelho et al. (2005). The fit, made assuming $[\alpha / \mathrm{Fe}]=+0.3$, is considerably improved.

\section{Limitations of the present libraries}

Atmospheric parameters of the stars. The effective temperature, surface gravity and metallicity of the stars in the libraries are essentially obtained from literature compilations. Beside dissimilar quality and occasional errors, these determinations results from various spectroscopic and photometric methods, based on different stellar models, and despite the efforts to reduce the inhomogeneity, the global consistency, from the spectroscopic determination of the atmospheric parameters to the computation of the isochrones in the plane of observed parameters still deserve to be improved.

Coverage of the parameter space. Lack of hot stars of low metallicity.

Abundance of individual elements. The libraries have the characteristic abundance pattern of the solar neighborhood, and therefore the models fail to reproduce the high metallicity populations with over-abundant $\mathrm{Mg}$, like elliptical galaxies or bulge globular clusters (see Fig. 2). The classical solution to overturn this limitation is to compute response functions of spectrophotometric indices to individual element (Korn et al. 2005). But this approach does not exploit the high spectral resolution of the libraries. 


\section{Next generation of models}

ELODIE.4. We have recently assembled a new version of the ELODIE library (version 3.1) where the data reduction and determination of the atmospheric parameters have been improved. The spectral range $(390-680 \mathrm{~nm})$ has also been extended to the blue limit of the spectrograph. To follow this version, we have selected from the ELODIE archive (Moultaka et al. 2004) all the stars present in the different libraries used for population synthesis and we also included stars from sparse regions of the parameters space. The new database counts about 5000 spectra for over 2000 stars. Using the ELODIE spectra at a resolution of $\mathrm{R}=42000$ will help to produce an homogeneous set of atmospheric parameters for a wide range of parameters, and provide measurements of individual abundances and rotation.

Semi-empirical library with $[\alpha / F e]$ resolution. To overstep the limitation due to the unresolved $[\alpha / \mathrm{Fe}]$ abundance of the libraries without loosing the advantage of the high spectral resolutions, the solutions may be (i) to build empirical libraries with variable $[\alpha / \mathrm{Fe}]$, (ii) to use theoretical libraries or (iii) to adopt a mixed approach where a theoretical library is used to correct an empirical one. The latter possibility offers the advantage to cover all the possible range of $[\alpha / \mathrm{Fe}]$ at any metallicity, which would not be reachable with a purely empirical library. This mixed approach avoids also the limitations of the theoretical libraries due to inaccuracy or incompleteness of line lists, oscillator strengths and atmosphere models. We present here a first attempt to generate such a semi-empirical library where each wavelength point of the grid based on the ELODIE library is differentially corrected to any $[\alpha / \mathrm{Fe}]$ using Coelho et al. (2005). It is assumed that the ELODIE library has the abundance pattern of the solar neighborhood.

In Fig. 2, we show that while the fit to low metallicity globular clusters with the standard Pegase.HR models is acceptable because the ELODIE library has a strong $[\alpha / \mathrm{Fe}]$ enhancement at low metallicity, the mismatch of the $\mathrm{Mg}$ becomes important for high metallicity clusters. Using the models built with the semi-empirical grid at an enhancement $[\alpha / \mathrm{Fe}] \approx+0.3$, the residuals are canceled.

Acknowledgements MC acknowledges the IAU financial support. PhP thanks FS.

\section{References}

Coelho, P., Barbuy, B., Meléndez, J., Schiavon, R. P., \& Castilho, B. V., 2005, A\&3A 443, 735

Fioc, M., \& Rocca-Volmerange, B., 1997, A\&A A 326, 950

Koleva, M., Bavouzet, N., Chilingarian, I., \& Prugniel, Ph, 2006, astro-ph/0602362

Korn, A. J., Maraston, C., \& Thomas, D., 2005, A\&A 438, 685

Le Borgne, D. et al., 2004, A\&\&A 425, 881

Ocvirk, P., Pichon, C., Lançon, A., \& Thiébaut, E., 2006, MNRAS 365, 74

Moultaka, J., Ilovaisky, S. A., Prugniel, P., \& Soubiran, C., 2004, PASP 116, 693

Prugniel, Ph. \& Soubiran, C.,2001, A\&A, 369, 1048

Prugniel, Ph. \& Soubiran, C., 2004, astro.ph-/0409214

Sánchez-Blázquez et al. 2006, MNRAS 371, 703

Schiavon, R., Caldwell, N., \& Rose, J., 2004, AJ 127, 1513

Schiavon, R., Rose, J., Courteau, S., \& McArthur, L., 2005, ApJS 160, 163

Valdes, F., Gupta, R., Rose, J., Singh, H., \& Bell, D., 2004, ApJS 152, 251

\section{Discussion}

Cid Fernandes: You are removing the continuum of your fits. is that because you do not want to worry about extinction or flux calibration issues? 
Prugniel: The shape of the continuum sensitive to several factors, and any small mismatch contributes considerably to the $\chi^{2}$. Being unsensitive to the continuum, our method is quite similar to spectrophotometric indices or equivalent widths... and is indeed not seriously affected by flux-calibration errors.

PeLeTIER: When you talk about interpolation in the library, are you thinking about a modern system of fitting functions?

Prugniel: We are fitting polynomials in Teff, $\log (\mathrm{g})$ and $\mathrm{Fe} / \mathrm{H}$ at each wavelength point to the whole set of spectra in the library. This allows to smooth the effects of the ignored parameters (like the stellar rotation), interpolate and generate the grid used for the population synthesis. The inversion of these polynomials allows to check the consistency of the input atmospheric parameters. It is like fitting functions.

PANTER: As well as you see similar residuals between Pegase.HR and Vazdekis/MILES, we see also the same residuals with MOPED analysis of SDSS galaxies and all models publically available. Do you really think that it is all the fault of the data? Could it not be that there is something missing from all the models?

PRUGNIEL: The residuals are similar when we compare the two high quality models, Pegase.HR and Vazdekis/MILES, but they are different from galaxy (or cluster) to galaxy, even though the $\mathrm{S} / \mathrm{N}$ is 200 or above. There are effect coming from the models, like the $\mathrm{Mg}$ or $\mathrm{CN}$ over-abundancy, but the main part of the residuals seems to be due to the data. 